\title{
p-Moment Stability of Stochastic Differential Delay Systems with Impulsive Jump and Markovian Switching
}

\author{
Lijun Gao \\ Department of Electrical Engineering and Automation, Qufu Normal University, Rizhao 276826, China \\ Correspondence should be addressed to Lijun Gao; gljwg1977@163.com
}

Received 2 February 2013; Revised 26 April 2013; Accepted 3 May 2013

Academic Editor: Yang Yi

Copyright (C) 2013 Lijun Gao. This is an open access article distributed under the Creative Commons Attribution License, which permits unrestricted use, distribution, and reproduction in any medium, provided the original work is properly cited.

\begin{abstract}
This paper investigates $p$-moment stability of the stochastic differential delay systems with impulsive jump and Markovian switching. Some stability criteria are obtained based on Lyapunov functional method and stochastic theory. It is shown that, even if all the subsystems governing the continuous dynamics without impulse are not stable, as impulsive and switching signal satisfies a dwell-time upper bound condition, impulses can stabilize the systems in the $p$-moment stability sense. The opposite situation is also developed for which all the subsystems governing the continuous dynamics are $p$-moment stable. The results can be easily applied to stochastic systems with arbitrarily large delays. The efficiency of the proposed results is illustrated by two numerical examples.
\end{abstract}

\section{Introduction}

Stochastic differential systems which include stochastic delay differential systems have attracted much attention, owing to stochastic modeling having played an important role in many ways such as science and engineering and forecast of the growth of population $[1,2]$. Stability analysis of different stochastic systems has been a subject of intense activities in the literature [3-9]. Switched systems are an important class of hybrid dynamical systems which are composed of a family of continuous time or discrete-time dynamical systems and a rule that orchestrates the switching among them [10]. A particular class of switched systems is named as markovian switched systems, whose system mode is governed by a Markov process. During the past few decades, many issues on Markovian switched systems such as stability and stabilization [11-14], $H_{\infty}$ control and filtering [15], and adaptive control problem $[16,17]$ have been well investigated.

Beside stochastic effects and markovian switching, impulsive effect likewise exists in many evolution processed in which systems states change abruptly at certain moments of time, involving such fields as biology, engineering, and information science [18]. Therefore, the stability investigation of stochastic differential systems with impulsive jump is interesting to many investigators. The $p$-moment stability of stochastic differential systems is studied in [19] for the systems with impulsive jump, nonswitched, and no time delay. Thus, the results in [19] cannot be easily applied to the class of impulsive systems with markovian switching and time-varying delay. Liu and Peng [20] discussed the $p$ moment stability of stochastic differential delay systems with impulsive jump and markovian switching. It is shown that when the delayed continuous dynamics are $p$-moment stable, the stability properties is not destroyed by impulse at discrete instants irrespective of the length of delay. It is also noticed that the conclusions received in [20] are restricted to the case that are all the subsystems that govern the continuous dynamics are stable in $p$-moment sense. There is no attention has been paid to the class of hybrid systems in which all the subsystems that govern the continuous dynamics is not stable. Thus, how to establish a sufficient condition of $p$-moment stability for the class of stochastic delays hybrid systems is the key problem to be solved in future research.

Based on the above analysis, in this work, the $p$-moment stability of stochastic differential delay systems with impulsive jump and markovian switching is investigated. Motivated by the work of [20], we first relax the global Lipschitz condition of impulsive control law $\Delta x\left(t_{k}\right)$. Then, we will propose some conditions of the $p$-moment stability for two classes of stochastic hybrid delay systems, that is, all the 
subsystems that govern the continuous dynamics are stable and not. Compared with [20], the criteria for the stable case in this paper are more general. Further, the results are applied to linear stochastic delay systems with arbitrarily large delays.

\section{Preliminaries}

Throughout this paper, unless otherwise specified, we let $\left(\Omega, F,\left\{F_{t}\right\}_{t \geq 0}, P\right)$ be a complete probability space with a filtration $\left\{F_{t}\right\}_{t \geq 0}$ satisfying the usual conditions, that is, it is right continuous and $F_{0}$ contains all $P$-null sets. $\omega(t)$ is an $m$ dimensional Brownian motion defined on $\left(\Omega, F,\left\{F_{t}\right\}_{t \geq 0}, P\right)$. Let $Z^{+}$define the set of nonnegative real numbers and $R^{n}$ the $n$-dimensional real Euclidean space. $|\cdot|$ denotes the Euclidean norm for vectors or the spectral norm for matrices. For $r>0$, let PC $\left([-r, 0], R^{n}\right)$ denote the class of functions from $[-r, 0]$ to $R^{n}$ satisfying the following: (i) it has at most a finite number of jump discontinuities on $(-r, 0]$; (ii) it is continuous from the right at all points in $[-r, 0)$. For simplicity, PC is used for $\mathrm{PC}\left([-r, 0], R^{n}\right)$ for the rest of this paper. For function $\phi$ : $[-r, 0] \rightarrow R^{n}$, a norm is defined as $\|\phi\|_{r}=\sup _{-r \leq \theta \leq 0}|\phi(\theta)|$. Given $x \in \operatorname{PC}\left([-r, \infty], R^{n}\right)$ and for each $t \in R^{+}$, define $x_{t}, x_{t^{-}} \in \mathrm{PC}$ by $x_{t}(s)=x(t+s)$ for $-r \leq s \leq 0$ and $x_{t^{-}}(s)=$ $x(t+s)$ for $-r \leq s<0$, respectively. Denote by $\mathrm{PC}_{F_{t}}^{p}$ the family of all $F_{t}$-measurable PC-valued random variables $\phi=\{\phi(\theta)$ : $-r \leq \theta \leq 0\}$, satisfying $\sup _{-r \leq \theta \leq 0} E\|\phi(\theta)\|^{p}<\infty$, where $E$ stands for the mathematical expectation. Let $\operatorname{PC}_{F_{t}}^{b}(\delta)=\{\phi$ : $\phi \in \mathrm{PC}_{F_{t}}^{p}\left([-r, 0], R^{n}\right)$ and $\left.\sup _{-r \leq \theta \leq 0} E\|\phi(\theta)\|^{p}<\delta\right\}$.

The Markov process $\{r(t), t \geq 0\}$ represents the switching between the different modes taking values in a finite state space $S=\{1,2, \ldots, N\}$ with generator $\pi=\left(\pi_{i j}\right)_{N \times N}$ given by

$$
\operatorname{Pr}\{r(t+\Delta)=j \mid r(t)=i\}= \begin{cases}\pi_{i j} \Delta+o(\Delta), & \text { if } i \neq j \\ 1+\pi_{i i} \Delta+o(\Delta), & \text { if } i=j\end{cases}
$$

where $\pi_{i j}$ is the transition rate from mode $i$ to $j$ and satisfies the following relations:

$$
\pi_{i j} \geq 0, \quad \pi_{i i}=-\sum_{j \neq i} \pi_{i j}
$$

and $o(\Delta)$ is such that $\lim _{\Delta \rightarrow 0} o(\Delta) / \Delta=0$. We assume that the Markov chain $r(t)$ is independent of the Brownian motion $\omega(\cdot)$. It is known that almost every sample path of $r(t)$ is a right-continuous step function with a finite number of simple jumps in any finite subinterval of $R^{+}=[0,+\infty)$.

Consider the following stochastic nonlinear delay system with impulsive jump and markovian switching

$$
\begin{aligned}
& d x(t)= f\left(x(t), x_{t}, t, r(t)\right) d t \\
&+g\left(x(t), x_{t}, t, r(t)\right) d \omega(t), \quad t \neq t_{k}, \\
& \Delta x(t)=I_{k}\left(x\left(t^{-}\right), t\right), \quad t=t_{k}, \\
& x\left(t_{0}+\theta\right)=\phi(\theta), \quad \theta \in[-r, 0],
\end{aligned}
$$

where $\phi(\theta) \in$ PC is the initial data, $x(t) \in R^{n}$ is the systems state, $x\left(t^{+}\right)$and $x\left(t^{-}\right)$denote the limit from the right and the left at point $t$, respectively, and $\left\{t_{k}: k \in Z^{+}\right\} \subset R^{+}$a strictly increasing sequence. We assume that for each $i \in S$, given functional $f: R^{n} \times \mathrm{PC} \times S \rightarrow R^{n}, g: R^{n} \times \mathrm{PC} \times S \rightarrow R^{n \times m}$ satisfying $f(0,0, t, i) \equiv g(0,0, t, i) \equiv 0 . I_{k}: R^{n} \times R \rightarrow R^{n}$ with $I_{k}(0, t) \equiv 0$ is the change of state variable at instant $t_{k}$. In this paper, we always assume that there exists a unique stochastic process satisfying systems (3), and all solutions of systems (3) are continuous on the right-hand side and on the left-hand side. Moreover, by $f(0,0, t, i) \equiv g(0,0, t, i) \equiv 0$ and $I_{k}(0, t) \equiv$ 0 , it is easily obtained that system (3) admits a trivial solution.

Definition 1 (see [20]). The trivial solution of system (3) is

(1) $p$-moment stable if for any initial data $x_{t_{0}}=\phi$ and any $\varepsilon>0$, there exists a $\delta>0$ such that

$$
E\|x(t)\|^{p}<\varepsilon, \quad t \geq t_{0},
$$

whenever $\|\phi\|_{r}^{p}<\delta$;

(2) uniformly $p$-moment stable if the $\delta$ in (1) is independent of $t_{0}$.

Definition 2. Let $C^{2,1}\left(R^{n} \times\left[t_{0}-r, \infty\right] \times S ; R^{+}\right)$denote the family of all nonnegative functions $V(x, t, i)$ that are continuously twice differentiable in $x$ and once differentiable with respect to $t$. For each $V(x, t, i) \in C^{2,1}\left(R^{n} \times\left[t_{0}-r, \infty\right] \times S ; R^{+}\right)$, define an operator $L V$ from $R^{n} \times\left[t_{0}-r, \infty\right] \times S$ to $R^{+}$as follows:

$$
\begin{aligned}
L V(x, t, i)= & V_{t}(x, t, i)+V_{x}(x, t, i) f(x, y, t, i) \\
& +\frac{1}{2} \operatorname{trace}\left[g^{T}(x, y, t, i) V_{x x}(x, t, i) g(x, y, t, i)\right] \\
& +\sum_{j=1}^{N} q_{i j} V(x, t, j),
\end{aligned}
$$

where

$$
\begin{gathered}
V_{t}(x, t, i)=\frac{\partial V(x, t, i)}{\partial t}, \\
V_{x}(x, t, i)=\left(\frac{\partial V(x, t, i)}{\partial x_{1}}, \ldots, \frac{\partial V(x, t, i)}{\partial x_{n}}\right), \\
V_{x x}(x, t, i)=\left(\frac{\partial^{2} V(x, t, i)}{\partial x_{i} \partial x_{j}}\right)_{n \times n} .
\end{gathered}
$$

Before giving the results, we need a lemma.

Lemma 3 (see [20]). If $V \in C^{2,1}\left(R^{n} \times\left[t_{0}-r, \infty\right] \times S ; R^{+}\right)$, then for any stopping times $0 \leq t_{1} \leq t_{2}<+\infty$,

$$
\begin{aligned}
E\left(V\left(x\left(t_{2}\right), t_{2}, r\left(t_{2}\right)\right)\right)= & E\left(V\left(x\left(t_{1}\right), t_{1}, r\left(t_{1}\right)\right)\right) \\
& +E\left(\int_{t_{1}}^{t_{2}} L V(x(s), s, r(s)) d s\right),
\end{aligned}
$$

as long as the integration involved exist and finite. 


\section{Main Results}

In this section, Lyapunov-based sufficient conditions for $p$ moment stability of system (3) are developed. The first result is concerned with $p$-moment stability of system (3), in the case when all the subsystems governing the continuous dynamics of (3) are stable. Intuitively, the conditions in the following theorem consist of three aspects: (i) the LyapunovKrasovskii functionals satisfy certain positive definite and decrescent conditions; (ii) there exist some negative estimates of the upper right-hand derivatives of the functionals with respect to each stable mode of system (3); (iii) the jumps induced by the impulses and the estimates on the decay rate of continuous dynamics satisfy certain conditions.

Theorem 4. Assume that there exist function $V(x(t), t, i) \in$ $C^{2,1}\left(R^{n} \times\left[t_{0}-r, \infty\right] \times S ; R^{+}\right)$and some positive constants $p, a$, $b$, $e_{i}$, the nonnegative and continuous functions $c_{i}(t)$ and $d_{i}(t)$, such that

(i) $a|x|^{p} \leq V(x(t), t, i) \leq b\left|x_{t}\right|_{r}^{p}$;

(ii) $E L V(x(t), t, i) \leq-c_{i}(t) E V(x(t), t, i)+d_{i}(t) E V\left(x_{t}, t, i\right)$, $t \in\left[t_{k-1}, t_{k}\right), k=1,2, \ldots$;

(iii) $E V\left(\phi(0)+I_{k}\left(\phi, t_{k}, \widetilde{i}\right) \leq e_{i} V\left(\phi(0), t_{k}^{-}, i\right)\right.$;

(iv) $0<c^{*}<1$, where $c^{*}=\sup \left\{c_{i}^{*} \mid c_{i}^{*}=b e_{i} / a, i=1\right.$, $2, \ldots\}$

(v) $c_{i}(t)>d_{i}(t) / c^{*}, t \in\left[t_{k}, t_{k+1}\right)$;

then the trivial solution of system (3) is uniformly p-moment stable.

Proof. For any $\varepsilon>0$, there exists a $\delta=\delta(\varepsilon)>0$ such that $\delta<\left(a c^{*} / b\right) \varepsilon$ independent of $t_{0}$. For any $t_{0} \geq 0$ and $x_{t_{0}}=\phi \epsilon$ $\mathrm{PC}_{F_{t}}^{b}(\delta)$, let $x(t)=x\left(t, t_{0}, \phi\right)$ be the solution of (3).

In the following, we will show that

$$
E V(x(t), t, i) \leq \frac{b}{c^{*}} \delta, \quad t \in\left[t_{k-1}, t_{k}\right), k \in Z^{+} .
$$

It will be firstly proved that

$$
E V(x(t), t, i) \leq \frac{b}{c^{*}} \delta, \quad t \in\left[t_{0}, t_{1}\right) .
$$

For any initial data $x_{t_{0}} \in \mathrm{PC}_{F_{t}}^{b}(\delta)$, it follows from assumption (i) that

$$
\begin{aligned}
E V(x(t), t, r(t)) & \leq b E\left\|x_{t_{0}}\right\|_{r}^{p} \\
& \leq b \delta<\frac{b}{c^{*}} \delta, \quad t \in\left[t_{0}-r, t_{0}\right] .
\end{aligned}
$$

Suppose that (9) is not true, then there exists some $t \in\left(t_{0}, t_{1}\right)$ such that

$$
E V(x(t), t, r(t))>\frac{b}{c^{*}} \delta>b \delta \geq E V\left(x\left(t_{0}\right), t_{0}, r\left(t_{0}\right)\right) .
$$

Set $t^{*}=\inf \left\{t \in\left[t_{0}, t_{1}\right): E V(x(t), t, r(t))>\left(b / c^{*}\right) \delta\right\}$. Observe that $E V(x(t), t, r(t))$ is continuous on $t \in\left[t_{0}, t_{1}\right)$, then $t^{*} \in$ $\left(t_{0}, t_{1}\right)$ and

$$
\begin{gathered}
E V\left(x\left(t^{*}\right), t^{*}, r\left(t^{*}\right)\right)=\frac{b}{c^{*}} \delta, \\
E V(x(t), t, r(t))<\frac{b}{c^{*}} \delta, \quad t \in\left[t_{0}-r, t^{*}\right) .
\end{gathered}
$$

In view of (10), define $t^{* *}=\sup \left\{t \in\left[t_{0}-r, t^{*}\right]:\right.$ $E V(x(t), t, r(t)) \leq b \delta\}$. Then $t^{* *} \in\left(t_{0}, t^{*}\right)$ and

$$
\begin{gathered}
E V\left(x\left(t^{* *}\right), t^{* *}, r\left(t^{* *}\right)\right)=b \delta, \\
E V(x(t), t, r(t))>b \delta, \quad t \in\left(t^{* *}, t^{*}\right] .
\end{gathered}
$$

Consequently, in view of (10)-(15), for all $t \in\left[t^{*}, t^{* *}\right]$ and $\theta \in[-r, 0]$, one has

$$
\begin{aligned}
E V( & x(t+\theta), t+\theta, r(t+\theta)) \\
& \leq \frac{b}{c^{*}} \delta \leq \frac{1}{c^{*}} E V\left(x\left(t^{*}\right), t^{*}, r\left(t^{*}\right)\right) \\
& \leq \frac{1}{c^{*}} E V(x(t), t, r(t)) .
\end{aligned}
$$

Now, combining (16) and condition (ii), we have

$$
\begin{aligned}
E L V & (x(t), t, r(t)) \\
& \leq-c_{i}(t) E V(x(t), t, r(t))+d_{i}(t) E V\left(x_{t}, t, r(t)\right) \\
& \leq\left(-c_{i}(t)+\frac{d_{i}(t)}{c^{*}}\right) E V(x(t), t, r(t)) .
\end{aligned}
$$

Applying Lemma 3, integrating (17) on $\left[t^{* *}, t^{*}\right]$, one obtains that

$$
\begin{aligned}
& E V\left(x\left(t^{*}\right), t^{*}, r\left(t^{*}\right)\right) \\
& \leq E V\left(x\left(t^{* *}\right), t^{* *}, r\left(t^{* *}\right)\right) \\
& \quad+\int_{t^{* *}}^{t^{*}}\left(-c_{i}(t)+\frac{d_{i}(t)}{c^{*}}\right) E V(x(s), s, r(s)) d s .
\end{aligned}
$$

By condition (v) and (14) and the Gronwall inequality, one has

$$
\begin{aligned}
E V( & \left.\left(t^{*}\right), t^{*}, r\left(t^{*}\right)\right) \\
& \leq E V\left(x\left(t^{* *}\right), t^{* *}, r\left(t^{* *}\right)\right) e^{\left(-c_{i}(t)+\left(d_{i}(t) / c^{*}\right)\right)\left(t^{*}-t^{* *}\right)} \\
\quad \leq & E V\left(x\left(t^{* *}\right), t^{* *}, r\left(t^{* *}\right)\right)=b \delta<\frac{b}{c^{*}} \delta .
\end{aligned}
$$

Since (19) contradicts with (12), inequality (9) holds and (8) is true for $k=1$.

Now, assume that

$$
E V(x(t), t, i) \leq \frac{b}{c^{*}} \delta, \quad t \in\left[t_{k-1}, t_{k}\right),
$$


for all $k \leq m$, where $k, m \in Z^{+}$. We proceed to show that

$$
E V(x(t), t, i) \leq \frac{b}{c^{*}} \delta, \quad t \in\left[t_{m}, t_{m+1}\right) .
$$

Suppose that (21) is not true, set $\bar{t}=\inf \left\{t \in\left[t_{m}, t_{m+1}\right)\right.$ : $\left.E V(x(t), t, r(t))>\left(b / c^{*}\right) \delta\right\}$. From condition (i), (iv), and (21), we know that

$$
\begin{aligned}
E V( & \left.x\left(t_{m}\right), t_{m}, r\left(t_{m}\right)\right) \\
& \leq e_{i_{m}} E V\left(x\left(t_{m}^{-}\right), t_{m}^{-}, r\left(t_{m}^{-}\right)\right) \\
& \leq b e_{i_{m}}\left\|x_{t_{m}^{-}}\right\|_{r}^{p} \\
& \leq \frac{b e_{i_{m}}}{a} \sup _{-r \leq \theta \leq 0} E V\left(x\left(t_{m}^{-}+\theta\right), t_{m}^{-}+\theta, r\left(t_{m}^{-}+\theta\right)\right) \\
& \leq \frac{b e_{i_{m}}}{a} \frac{b}{c^{*}} \delta \leq b \delta<\frac{b}{c^{*}} \delta .
\end{aligned}
$$

Owing to $E V(x(t), t, r(t))$ is continuous on $t \in\left[t_{m}, t_{m+1}\right)$, then $\bar{t} \in\left(t_{m}, t_{m+1}\right)$ and

$$
\begin{gathered}
E V(x(\bar{t}), \bar{t}, r(\bar{t}))=\frac{b}{c^{*}} \delta, \\
E V(x(t), t, r(t))<\frac{b}{c^{*}} \delta, \quad t \in\left[t_{m}, \bar{t}\right) .
\end{gathered}
$$

Define $\underline{t}=\sup \left\{t \in\left[t_{0}-r, \bar{t}\right]: E V(x(t), t, r(t)) \leq b \delta\right\}$, then $\underline{t} \in\left(t_{m}, \overline{\bar{t}}\right)$ and

$$
\begin{gathered}
E V(x(\underline{t}), \underline{t}, r(\underline{t}))=b \delta, \\
E V(x(t), t, r(t))>b \delta, \quad t \in(\underline{t}, \bar{t}] .
\end{gathered}
$$

Fix any $t \in[t, \bar{t}]$, when $t+\theta \geq t_{m}$ for all $\theta \in[-r, 0]$, then from (22)-(26), one has

$$
\begin{aligned}
E V( & x(t+\theta), t+\theta, r(t+\theta)) \\
& \leq \frac{b}{c^{*}} \delta \leq \frac{1}{c^{*}} E V(x(\bar{t}), \bar{t}, r(\bar{t})) \\
& \leq \frac{1}{c^{*}} E V(x(t), t, r(t)) .
\end{aligned}
$$

When $t+\theta<t_{m}$ for some $\theta \in[-r, 0]$, without loss of generality, we assume that $t+\theta \in\left[t_{l-1}, t_{l}\right)$ for some $l \in Z^{+}$, $l<m$, then from (20) and (25), we obtain that

$$
\begin{aligned}
E V( & x(t+\theta), t+\theta, r(t+\theta)) \\
& \leq \frac{b}{c^{*}} \delta \leq \frac{1}{c^{*}} E V(x(\bar{t}), \bar{t}, r(\bar{t})) \\
& \leq \frac{1}{c^{*}} E V(x(t), t, r(t)) .
\end{aligned}
$$

Therefore, from condition (ii), (27), and (28), one gets

$$
\begin{aligned}
E L V & (x(t+\theta), t+\theta, r(t+\theta)) \\
& \leq\left(-c_{i_{m}}(t)+\frac{d_{i_{m}}(t)}{c^{*}}\right) E V(x(t), t, r(t)) .
\end{aligned}
$$

Similar to the argument on $\left[t^{* *}, t^{*}\right]$, an application of Ito's formula on $[\underline{t}, \bar{t}]$ will lead to $E V(x(\bar{t}), \bar{t}, r(\bar{t}))<\left(b / c^{*}\right) \delta$, which would contradict with (23). So the inequality (21) is true. Therefore, by mathematical induction, one can obtain that (8) holds for all $k \in Z^{+}$. Then from condition (i) and the definition of $\delta$, we have

$$
E\|x(t)\|^{p} \leq \frac{b}{a c^{*}} \delta<\varepsilon, \quad t \geq t_{0} .
$$

According to Definition 1, it is concluded that the trivial solution of system (3) is uniformly $p$-moment stable. The proof is complete.

Corollary 5. Assume that there exist function $V(x(t), t, i) \in$ $C^{2,1}\left(R^{n} \times\left[t_{0}-r, \infty\right] \times S ; R^{+}\right)$and some positive constants $p, a$, $b, e_{i}, c_{i}$, and $d_{i}$ such that

(i) $a|x|^{p} \leq V(x(t), t, i) \leq b\left|x_{t}\right|_{r}^{p}$;

(ii) $E L V(x(t), t, i) \leq-c_{i} E V(x(t), t, i)+d_{i} E V\left(x_{t}, t, i\right), t \in$ $\left[t_{k-1}, t_{k}\right), k=1,2, \ldots ;$

(iii) $E V\left(\phi(0)+I_{k}\left(\phi, t_{k}, \widetilde{i}\right) \leq e_{i} V\left(\phi(0), t_{k}^{-}, i\right)\right.$;

(iv) $0<c^{*}<1$, where $c^{*}=\sup \left\{c_{i}^{*} \mid c_{i}^{*}=\left(b e_{i} / a\right), i=\right.$ $1,2, \ldots\}$

(v) $c_{i}>d_{i} / c^{*}, t \in\left[t_{k}, t_{k+1}\right)$;

then the trivial solution of system (3) is uniformly p-moment stable.

Proof. Replacing $c_{i}(t)$ and $d_{i}(t)$ in Theorem 4 with $c_{i}$ and $d_{i}$, respectively, we find that it is a direct conclusion of Theorem 4.

Remark 6. From condition (ii) in Theorem 4, it is seen that each of the continuous dynamics is stable. On the other hand, from condition (iv), we see that each of the discrete dynamics is also stabilizing $\left(0<c^{*}<1\right.$ implies $\left.e_{i}<1\right)$. It implies that the stability properties of a time-delay impulsive markovian switched system with stable continuous dynamics can be preserved under stabilizing impulsive perturbations irrespective of the times of impulses and switching. Hence, the bound of dwell-time is not necessary.

Remark 7. If condition (iii) is omitted in Corollary 5, the result is consistent with that of Theorem 1 in [20]. Thus, the results in this work are an extension of that in [20].

We proceed to consider in next the $p$-moment stability of systems (3). It is supposed that all the subsystems governing the continuous dynamics of (3) can be unstable while the impulses are stabilizing. Intuitively, the conditions in the following theorem consist of four aspects: (i) the Lyapunov functionals satisfy certain positive definite and decrescent conditions; (ii) there exist some positive estimates of the upper right-hand derivatives of the functionals with respect to each unstable mode of system (3); (iii) the dwell time of each mode of system (3) satisfies some supper bounds; (iv) the jumps induced by the stabilizing impulses satisfy certain diminishing conditions. 
Theorem 8. Assume that there exist function $V(x(t), t, i) \in$ $C^{2,1}\left(R^{n} \times\left[t_{0}-r, \infty\right] \times S ; R^{+}\right)$and some positive scalars $p, a, b$, $e_{i}, \rho<1, \alpha$, and $\beta$ such that

(i) $a|x|^{p} \leq V(x(t), t, i) \leq b\left|x_{t}\right|_{r}^{p}$;

(ii) $E V\left(\phi(0)+I_{k}\left(\phi, t_{k}, \widetilde{i}\right) \leq \rho e_{i} V\left(\phi(0), t_{k}^{-}, i\right)\right.$;

(iii) $0<c^{*}<1$, where $c^{*}=\sup \left\{c_{i}^{*} \mid c_{i}^{*}=(b / a) \rho e_{i}, i=\right.$ $1,2, \ldots\}$

(iv) there exist nonnegative and piecewise continuous functions $c_{i}(t):\left[t_{0}, \infty\right) \rightarrow R^{n}$ satisfying $\int_{t}^{t+\alpha} c_{i}(s) d s \leq \alpha \beta$ for all $t \geq t_{0}$, such that

$$
\begin{array}{r}
E L V(x(t), t, i) \leq c_{i}(t) E V(x(t), t, i), \\
t \in\left[t_{k-1}, t_{k}\right), \quad k=1,2, \ldots,
\end{array}
$$

whenever $t \geq t_{0}$ and $\phi \in \mathrm{PC}_{F_{t}}^{b}$ are such that ${ }^{*} E L V$ $(\phi(s), t+s, \widetilde{i}) \leq E V(\phi(0), t, i) ;$

(v) $\sup _{k \in Z^{+}}\left\{t_{k}-t_{k-1}\right\}=\alpha<-(1 / \beta) \ln \left((b / a) \rho e_{i}\right), k \in Z^{+}$; then the trivial solution of system (3) is uniformly pmoment stable.

Proof. For any $\varepsilon>0$, there exists a $\delta=\delta(\varepsilon)>0$ such that $\delta<\left(a c^{*} / b\right) \varepsilon$ independent of $t_{0}$. For any $t_{0} \geq 0$ and $x_{t_{0}}=\phi \epsilon$ $\mathrm{PC}_{F_{t}}^{b}(\delta)$, let $x(t)=x\left(t, t_{0}, \phi\right)$ be the solution of (3).

By generalized Ito's formula (Lemma 3), when $t \in\left[t_{k}\right.$, $\left.t_{k+1}\right)$, we get

$$
\begin{aligned}
E V(x(t), t, r(t)) \\
=E V\left(x\left(t_{k}\right), t_{k}, r\left(t_{k}\right)\right) \\
\quad+E\left(\int_{t_{k}}^{t} L V(x(s), s, r(s)) d s\right) \\
\quad+E\left(\int_{t_{k}}^{t} \frac{\partial V(x(s), s, r(s))}{\partial x(s)} g(x(s), s, r(s)) d \omega(s)\right) \\
=E V\left(x\left(t_{k}\right), t_{k}, r\left(t_{k}\right)\right) \\
\quad+E\left(\int_{t_{k}}^{t} L V(x(s), s, r(s)) d s\right) .
\end{aligned}
$$

Given small enough $\Delta t>0$ such that $t+\Delta t \in\left[t_{k}, t_{k+1}\right)$, one has

$$
\begin{array}{r}
E V(x(t+\Delta t), t+\Delta t, r(t+\Delta t)) \\
=E V\left(x\left(t_{k}\right), t_{k}, r\left(t_{k}\right)\right) \\
+E\left(\int_{t_{k}}^{t+\Delta t} L V(x(s), s, r(s)) d s\right), \\
t \in\left[t_{k}, t_{k+1}\right) .
\end{array}
$$

In view of condition (iv), (32), and (33), it follows that

$$
\begin{aligned}
& E V(x(t+\Delta t), t+\Delta t, r(t+\Delta t))-E V(x(t), t, r(t)) \\
&= \int_{t}^{t+\Delta t} E L V(x(s), s, r(s)) d s \\
& \leq \int_{t}^{t+\Delta t} c_{i}(t) E V(x(s), s, r(s)) d s, \\
& t \in\left[t_{k}, t_{k+1}\right) .
\end{aligned}
$$

From (34), one gets

$$
\begin{array}{r}
D^{+} E V(x(t), t, r(t)) \leq c_{i}(t) E V(x(t), t, r(t)), \\
t \in\left[t_{k}, t_{k+1}\right),
\end{array}
$$

where $D^{+}$is the Dini-derivative defined as

$$
D^{+} V(t)=\lim _{h \rightarrow 0^{+}} \sup \frac{V(t+h)-V(t)}{h},
$$

in which $V(t)$ is continuous function.

In the following, we will show that

$$
E V(x(t), t, i) \leq \frac{b}{c^{*}} \delta, \quad t \in\left[t_{k-1}, t_{k}\right), k \in Z^{+} .
$$

For this purpose, we first prove that

$$
E V(x(t), t, i) \leq \frac{b}{c^{*}} \delta, \quad t \in\left[t_{0}, t_{1}\right) .
$$

From condition (i) and $x_{t_{0}} \in \mathrm{PC}_{F_{t}}^{b}(\delta)$, it follows that

$$
\begin{aligned}
E V(x(t), t, r(t)) & \leq b E\left\|x_{t_{0}}\right\|_{r}^{p} \\
& \leq b \delta<\frac{b}{c^{*}} \delta, \quad t \in\left[t_{0}-r, t_{0}\right] .
\end{aligned}
$$

If (38) is not true, there must exist some $t \in\left[t_{0}, t_{1}\right)$ such that

$$
E V(x(t), t, r(t))>\frac{b}{c^{*}} \delta>b \delta \geq E V\left(x\left(t_{0}\right), t_{0}, r\left(t_{0}\right)\right) .
$$

Let $t^{*}=\inf \left\{t \in\left[t_{0}, t_{1}\right): E V(x(t), t, r(t))>\left(b / c^{*}\right) \delta\right\}$. From (39), one has $t^{*} \in\left(t_{0}, t_{1}\right)$ and

$$
\begin{gathered}
E V\left(x\left(t^{*}\right), t^{*}, r\left(t^{*}\right)\right)=\frac{b}{c^{*}} \delta, \\
E V(x(t), t, r(t))<\frac{b}{c^{*}} \delta, \quad t \in\left[t_{0}-r, t^{*}\right), \\
D^{+} E V\left(x\left(t^{*}\right), t^{*}, r\left(t^{*}\right)\right) \geq 0 .
\end{gathered}
$$

Owing to $\left(b / c^{*}\right) \delta>b \delta$ and $E V(x(t), t, r(t)) \leq b \delta$ for $t \in$ $\left[t_{0}-r, t_{0}\right]$, then there exists a $t^{* *} \in\left[t_{0}, t^{*}\right)$ such that

$$
\begin{gathered}
E V\left(x\left(t^{* *}\right), t^{* *}, r\left(t^{* *}\right)\right)=b \delta, \\
E V(x(t), t, r(t))>b \delta, \quad t \in\left(t^{* *}, t^{*}\right], \\
D^{+} E V\left(x\left(t^{*}\right), t^{*}, r\left(t^{*}\right)\right) \geq 0 .
\end{gathered}
$$


Consequently, by (41) and (42), for $t \in\left[t^{*}, t^{* *}\right]$, we have

$$
E V(x(t+s), t+s, r(t+s)) \leq \frac{b \delta}{c^{*}} \leq \frac{1}{c^{*}} E V(x(t), t, r(t)) \text {. }
$$

So, for $t \in\left[t^{*}, t^{* *}\right]$, it has

$$
D^{+} E V(x(t), t, r(t)) \leq c_{i}(t) E V(x(t), t, r(t)) .
$$

Integrating (44) from $t^{* *}$ to $t^{*}$

$$
\int_{t^{* *}}^{t^{*}} \frac{D^{+} E V(x(s), s, r(s))}{E V(x(s), s, r(s))} d s \leq \int_{t_{0}}^{t_{1}} c_{i}(t) d s \leq \alpha \beta .
$$

Meanwhile, by condition (v), one has

$$
\begin{aligned}
\int_{t^{* *}}^{t^{*}} \frac{D^{+} E V(x(s), s, r(s))}{E V(x(s), s, r(s))} d s \\
\quad=\int_{E V\left(x\left(t^{* *}\right), t^{* *}, r\left(t^{* *}\right)\right)}^{E V\left(x\left(t^{*}\right), t^{*}, r\left(t^{*}\right)\right)} \frac{1}{u} d u \\
\quad=\int_{b \delta}^{\left(b / c^{*}\right) \delta} \frac{1}{u} d u=-\ln c^{*}>c_{i}\left(t_{1}-t_{0}\right),
\end{aligned}
$$

which is contradictory with (45), so (38) is true.

By condition (i) and (38), we have

$$
\begin{aligned}
E V( & \left.x\left(t_{1}\right), t_{1}, r\left(t_{1}\right)\right) \\
& \leq \rho e_{i_{1}} E V\left(x\left(t_{1}^{-}\right), t_{1}^{-}, r\left(t_{1}^{-}\right)\right) \\
& \leq b e_{i_{1}}\left\|x_{t_{1}^{-}}\right\|_{r}^{p} \\
& \leq \frac{b}{a} \rho e_{i_{1}} \sup _{-r \leq \theta \leq 0} E V\left(x\left(t_{1}^{-}+\theta\right), t_{1}^{-}+\theta, r\left(t_{1}^{-}+\theta\right)\right) \\
& \leq \frac{b}{a} \frac{b}{c^{*}} \rho e_{i_{1}} \delta \leq b \delta<\frac{b}{c^{*}} \delta .
\end{aligned}
$$

Now, we assume that (37) holds for $k=1,2, \ldots, m$, that is,

$$
E V(x(t), t, r(t)) \leq \frac{b}{c^{*}} \delta, \quad t \in\left[t_{k-1}, t_{k}\right] .
$$

We will prove that, for $k=m+1$,

$$
E V(x(t), t, r(t)) \leq \frac{b}{c^{*}} \delta, \quad t \in\left[t_{m}, t_{m+1}\right) .
$$

Suppose that (49) does not hold, by the same procedure as in $\left[t_{0}, t_{1}\right)$, we can get a contradiction and (49) follows. Finally, we get

$$
E\|x(t)\|^{p} \leq \frac{b}{a c^{*}} \delta<\varepsilon, \quad t \geq t_{0} .
$$

From Definition 1, it is concluded that the trivial solution of system (3) is uniformly $p$-moment stable. The proof is complete.
Corollary 9. Assume that there exist positive scalars $p, a, b$, $\rho<1, e_{i}, c_{i}(i \in S), \alpha$, and $\beta$ such that conditions (iv) in Theorem 8 are replaced by the following:

(iv) ${ }^{*}$ there exists positive number $c_{i}$ such that

$$
\begin{array}{r}
E L V(x(t), t, i) \leq c_{i} E V(x(t), t, i), \\
t \in\left[t_{k-1}, t_{k}\right), \quad k=1,2, \ldots,
\end{array}
$$

whenever $t \geq t_{0}$ and $\varphi \in \operatorname{PC}_{F_{t}}^{b}\left([-r, 0] ; R^{n}\right)$ are such that $c^{*} E L V(\varphi(s), t+s, \widetilde{i}) \leq E V(\varphi(0), t, i) ;$ and all other conditions remain the same, then the trivial solution of system (3) is uniformly p-moment stable.

Proof. Similar to the procedure of Theorem 8, correspondingly, the item $c_{i}(t)$ in the proof is replaced by $c_{i}$.

Remark 10. It is clear that Theorem 8 implies that each of the continuous dynamics can be unstable, since the item $c_{i}(t)$ in condition (iv), which characterizes the changing rate of Lyapunov function $V(x(t), t, r(t))$ at $t$, is assumed to be nonnegative. Theorem 8 shows that an unstable stochastic delay system can be successfully stabilized by impulse.

Remark 11. Constant $e_{i}$ in condition (ii) characterizes certain perturbations in the overall impulsive stabilization process, that is, it is not strictly required by Theorem 8 that each impulse contributes to stabilize the system, as long as the overall contribution of the impulses is stabilizing. When $e_{i} \equiv$ 1 , it is easily obtained that each impulse must be stabilizing $(\rho<1)$, which is more restrictive.

Now consider the linear stochastic system with impulses and markovian switching

$$
\begin{gathered}
d x(t)=[A(r(t)) x(t)+B(r(t)) x(t-r)] d t \\
+[C(r(t)) x(t)+D(r(t)) x(t-r)] d \omega(t), \\
t \neq t_{k}, \\
\Delta x(t)=E_{k}\left(x\left(t^{-}\right), r(t)\right), \quad t=t_{k}, \\
x\left(t_{0}+\theta\right)=\phi(\theta), \quad \theta \in[-r, 0],
\end{gathered}
$$

where $A_{i}, B_{i}, C_{i}, D_{i}$, and $E_{k}$ are all $n \times n$ matrices and $\omega$ is a one-dimensional standard wiener process. $r(t)$ is a Markov chain taking values in $S=\{1,2, \ldots, N\}$ in which the transition rate is $\pi_{i j}$.

Theorem 12. When $r(t)=i \in S$, define $A(r(t))=A_{i}$, $B(r(t))=B_{i}, C(r(t))=C_{i}, D(r(t))=D_{i}$.

(i) If there exist constants $p_{i}, c_{i}, d_{i}, \varepsilon_{1}$, and $\varepsilon_{2}$ such that

$$
p_{i} \lambda_{\max }\left(A_{i}^{T}+A_{i}+\varepsilon_{1} I\right)+p_{i}\left(1+\varepsilon_{2}\right)\left\|D_{i}\right\|^{2}+\sum_{j=1}^{N} \pi_{i j} p_{j}
$$




$$
\begin{gathered}
\leq-c_{i}, p_{i}\left\{\varepsilon_{1}^{-1}\left\|B_{i}\right\|^{2}+\left(1+\varepsilon_{2}^{-1}\right)\left\|G_{i}\right\|^{2}\right\} \leq d_{i} ; \\
p_{i} c_{i}\left\|I+E_{k}\right\|^{2}>d_{i},
\end{gathered}
$$

then system (52) is uniformly p-moment stable.

(ii) If there exist constants $c_{i}$, $\varepsilon_{1}$, and $\varepsilon_{2}$ such that

$$
\begin{aligned}
& p_{i} \lambda_{\max }\left(A_{i}^{T}+A_{i}+\varepsilon_{1} I\right)+p_{i}\left(1+\varepsilon_{2}\right)\left\|D_{i}\right\|^{2} \\
&+ \sum_{j=1}^{N} \pi_{i j} p_{j}+2 p_{i} \ln \left\|I+E_{k}\right\| \\
& \times\left[\varepsilon_{1}^{-1}\left\|B_{i}\right\|^{2}+\left(1+\varepsilon_{2}^{-1}\right)\left\|G_{i}\right\|^{2}\right] \leq c_{i} ; \\
& \ln \left(p_{i}\left\|I+E_{k}\right\|\right) \leq-\frac{1}{2} c_{i}\left(t_{k+1}-t_{k}\right),
\end{aligned}
$$

then system (52) is uniformly p-moment stable.

Proof. It is a direct consequence of Corollaries 5 and 9 with $V(x(t), r(t))=p_{i}\|x(t)\|^{2}$.

\section{Numerical Examples}

The applicability of the results derived in the preceding section is illustrated by the following two examples.

Example 1. Consider the following linear impulsive switching delay system:

$$
\begin{aligned}
d x(t)= & {[A(r(t)) x(t)+B(r(t)) x(t-1)] d t } \\
& +[C(r(t)) x(t)+D(r(t)) x(t-1)] d \omega(t), \\
& t \neq t_{k}, \\
& \Delta x(t)=E_{k}\left(x\left(t^{-}\right), r(t)\right), \quad t=t_{k},
\end{aligned}
$$

where

$$
\begin{array}{ccc}
A_{1}= & {\left[\begin{array}{ccc}
0.1 & 0.2 & -0.1 \\
0.2 & 0.15 & 0.3 \\
0 & 0.24 & 0.1
\end{array}\right],} & B_{1}=\left[\begin{array}{ccc}
-0.24 & 0.04 & 0 \\
0.24 & -0.4 & 0.1 \\
0 & 0.24 & -0.2
\end{array}\right], \\
D_{1}=\left[\begin{array}{ccc}
0.5 & 0.4 & 0.2 \\
-0.24 & 0.32 & 0.3 \\
0.6 & 0.26 & -0.5
\end{array}\right], & G_{1}=\left[\begin{array}{ccc}
0.14 & 0 & -0.21 \\
0.2 & 0.1 & 0.16 \\
0.1 & 0.15 & 0.17
\end{array}\right], \\
A_{2}=\left[\begin{array}{ccc}
0.2 & 0.3 & -0.1 \\
0.3 & 0.1 & 0.3 \\
0 & 0.2 & 0.1
\end{array}\right], & B_{2}=\left[\begin{array}{ccc}
-0.2 & 0.04 & 0 \\
0.2 & -0.4 & 0.1 \\
0 & 0.2 & -0.2
\end{array}\right], \\
D_{2}=\left[\begin{array}{ccc}
0.5 & 0.4 & 0.2 \\
-0.2 & 0.3 & 0.3 \\
0.6 & 0.2 & -0.5
\end{array}\right], & G_{2}=\left[\begin{array}{ccc}
0.24 & 0 & -0.21 \\
0.2 & 0.1 & 0.16 \\
0.1 & 0.1 & 0.17
\end{array}\right], \\
E_{k}=\left[\begin{array}{ccc}
-0.5 & 0 \\
0 & -0.4 & 0 \\
0 & 0 & -0.3
\end{array}\right], & \pi=\left[\begin{array}{cc}
-\frac{1}{3} & \frac{1}{3} \\
2 & 2 \\
\frac{2}{3} & -\frac{2}{3}
\end{array}\right]
\end{array}
$$

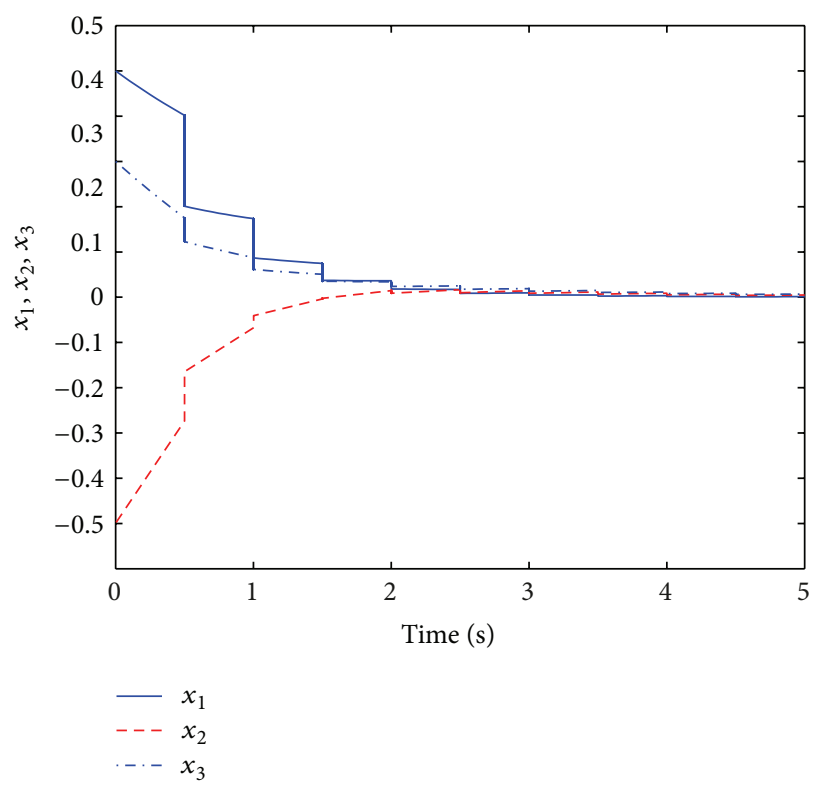

FIGURE 1: State response of system (55) under impulsive perturbations.

Set $\varepsilon_{1}=\varepsilon_{2}=0.5, p_{i}=1$. It is easy to see that $\lambda_{\max }\left(A_{1}^{T}+\right.$ $\left.A_{1}+0.5 I\right)=1.3819, \lambda_{\max }\left(A_{2}^{T}+A_{2}+0.5 I\right)=1.5020,\left\|B_{1}\right\|^{2}=$ 0.3141, $\left\|B_{2}\right\|^{2}=0.2764,\left\|D_{1}\right\|^{2}=0.9175,\left\|D_{2}\right\|^{2}=0.8737$, $\left\|G_{1}\right\|^{2}=0.1360,\left\|G_{2}\right\|^{2}=0.1219$, and $\left\|I+E_{k}\right\|^{2}=4.9$. Taking $c_{1}=3.1, c_{2}=3.2, t_{k}-t_{k-1}=0.22$, we can verify that all the conditions (ii) of Theorem 12 are satisfied:

$$
\begin{aligned}
& \lambda_{\max }(\left.A_{1}^{T}+A_{1}+\varepsilon_{1} I\right)+\left(1+\varepsilon_{2}\right)\left\|D_{1}\right\|^{2} \\
&+\sum_{j=1}^{N} \pi_{1 j}+2 \ln \left\|I+E_{k}\right\| \\
& \times\left[\varepsilon_{1}^{-1}\left\|B_{1}\right\|^{2}+\left(1+\varepsilon_{2}^{-1}\right)\left\|G_{1}\right\|^{2}\right] \\
&= 3.0190 \leq c_{1}=3.1 ; \\
& \lambda_{\max }\left(A_{2}^{T}+A_{2}+\varepsilon_{1} I\right)+\left(1+\varepsilon_{2}\right)\left\|D_{1}\right\|^{2} \\
&+\sum_{j=1}^{N} \pi_{2 j}+2 \ln \left\|I+E_{k}\right\| \\
& \quad \times\left[\varepsilon_{1}^{-1}\left\|B_{2}\right\|^{2}+\left(1+\varepsilon_{2}^{-1}\right)\left\|G_{2}\right\|^{2}\right] \\
&=3.1574 \leq c_{2}=3.2 ; \\
& \ln \left\|I+E_{k}\right\|^{2}=-0.7133 \leq-c_{i}\left(t_{k+1}-t_{k}\right) \\
&=-3.2 \times 0.22=-0.7040 .
\end{aligned}
$$

So, system (55) is $p$-moment stable. Numerical simulations for this example are shown in Figures 1 and 2. It is clearly demonstrated that impulses can successfully stabilize an otherwise unstable stochastic delay system. 


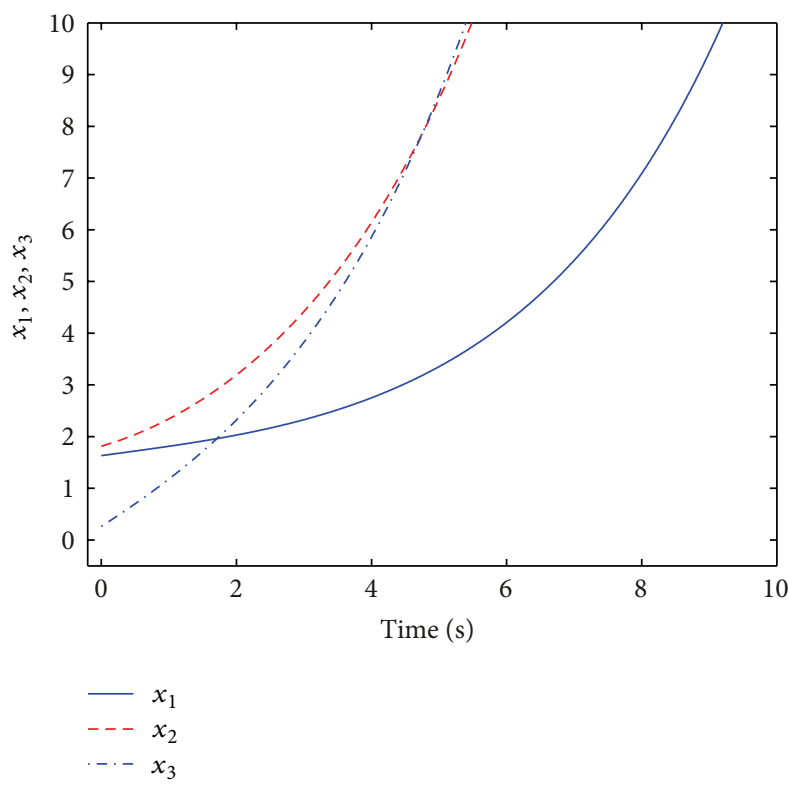

(a)

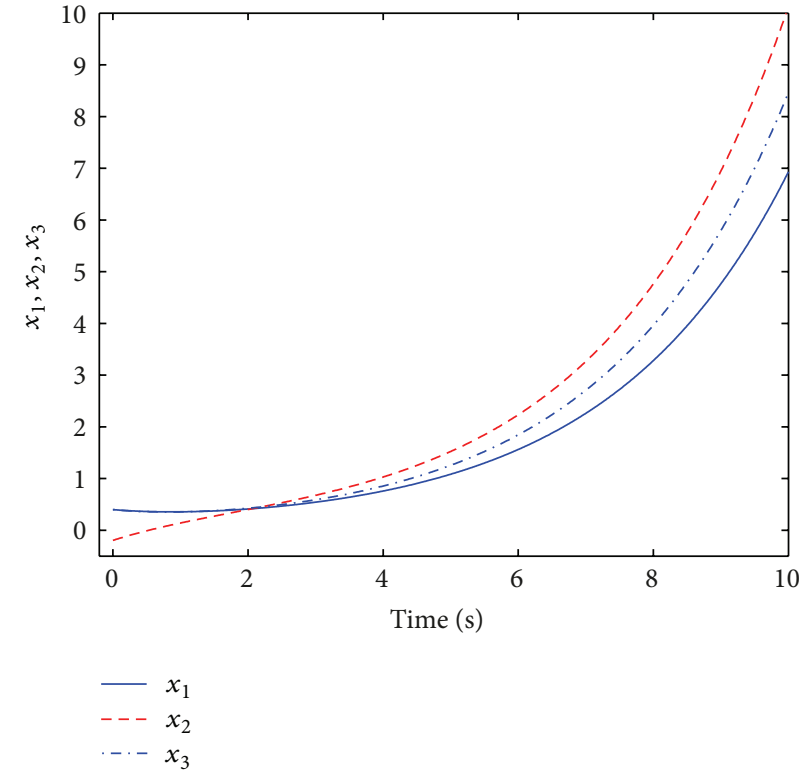

(b)

FIGURE 2: State response of system (55) without impulsive perturbations.

Example 2. Consider the following linear impulsive switching delay system:

$$
\begin{aligned}
d x(t)= & {[A(r(t)) x(t)+B(r(t)) x(t-1)] d t } \\
& +[C(r(t)) x(t)+D(r(t)) x(t-1)] d \omega(t), \\
& t \neq t_{k}, \\
& \Delta x(t)=E_{k}\left(x\left(t^{-}\right), r(t)\right), \quad t=t_{k},
\end{aligned}
$$

where

$$
\begin{gathered}
A_{1}=\left[\begin{array}{cc}
-4 & 0 \\
0 & -5
\end{array}\right], \quad B_{1}=\left[\begin{array}{ll}
0.2 & 0.3 \\
0.5 & 0.1
\end{array}\right], \\
D_{1}=\left[\begin{array}{ll}
0.3 & 0.4 \\
0.5 & 0.2
\end{array}\right], \quad G_{1}=\left[\begin{array}{ll}
0.8 & 0.1 \\
0.1 & 0.3
\end{array}\right], \\
E_{k}=\left[\begin{array}{cc}
-0.6 & 0.5 \\
0.3 & -0.5
\end{array}\right], \\
A_{2}=\left[\begin{array}{cc}
-3 & 0 \\
0 & -4
\end{array}\right], \quad B_{2}=\left[\begin{array}{ll}
0.3 & 0.5 \\
0.4 & 0.3
\end{array}\right], \\
D_{2}=\left[\begin{array}{cc}
0.5 & 0.1 \\
0.4 & 0.1
\end{array}\right], \quad G_{2}=\left[\begin{array}{ll}
0.2 & 0.2 \\
0.1 & 0.4
\end{array}\right], \\
P=\left[\begin{array}{ll}
0.5 & 0.5 \\
0.5 & 0.5
\end{array}\right] .
\end{gathered}
$$

Choosing $\varepsilon_{1}=\varepsilon_{2}=2, p_{i}=1$. It is easy to see that $\lambda_{\text {max }}\left(A_{1}^{T}+\right.$ $\left.A_{1}+2 I\right)=-6, \lambda_{\text {max }}\left(A_{2}^{T}+A_{2}+2 I\right)=-4,\left\|B_{1}\right\|^{2}=0.3403$, $\left\|B_{2}\right\|^{2}=0.5687,\left\|D_{1}\right\|^{2}=0.5009,\left\|D_{2}\right\|^{2}=0.4298,\left\|G_{1}\right\|^{2}=$ 0.6712, $\left\|G_{2}\right\|^{2}=0.2347,\left\|I+E_{k}\right\|^{2}=0.7467$. Taking $c_{1}=3.3$, $c_{2}=1.6, d_{1}=1.2, d_{2}=0.65$, we can verify that all the conditions (i) of Theorem 12 are satisfied:

$$
\begin{gathered}
\lambda_{\max }\left(A_{i}^{T}+A_{i}+\varepsilon_{1} I\right)+\left(1+\varepsilon_{2}\right)\left\|D_{i}\right\|^{2} \\
+\sum_{j=1}^{N} \pi_{i j}=\left\{\begin{array}{l}
-3.4973<-3.3=c_{1} ; \\
-1.7166<-1.6=c_{2} ;
\end{array}\right. \\
\varepsilon_{1}^{-1}\left\|B_{i}\right\|^{2}+\left(1+\varepsilon_{2}^{-1}\right)\left\|G_{i}\right\|^{2}=\left\{\begin{array}{l}
1.1770<1.2=d_{1} ; \\
0.6364<0.65=d_{2} ;
\end{array}\right. \\
\left\|I+E_{k}\right\|^{2} c_{i}=0.7467 \times\left\{\begin{array}{l}
3.3=2.4641>1.2=d_{1} ; \\
1.6=1.1947>0.65=d_{2} .
\end{array}\right.
\end{gathered}
$$

Theorem 12 guarantees that the trivial solution of system (58) is $p$-moment stable. Numerical simulations are shown in Figure 3. It is clearly demonstrated that the $p$-moment stability properties can be preserved irrespective of the length of the delay.

\section{Conclusions}

In this paper, a method of multiple Lyapunov functionals has been applied to deal with the effects of impulses on $p$ moment stability of stochastic differential delay systems with impulsive jump and markovian switching. Some stability criteria are obtained based on Lyapunov functional method and stochastic theory. It is shown that, even if all the subsystems governing the continuous dynamics without impulse are not stable, as impulsive and switching signal satisfies a dwell-time upper bound condition, impulses can stabilize the systems in the $p$-moment stability sense. The opposite situation is also developed for which all the subsystems governing the 


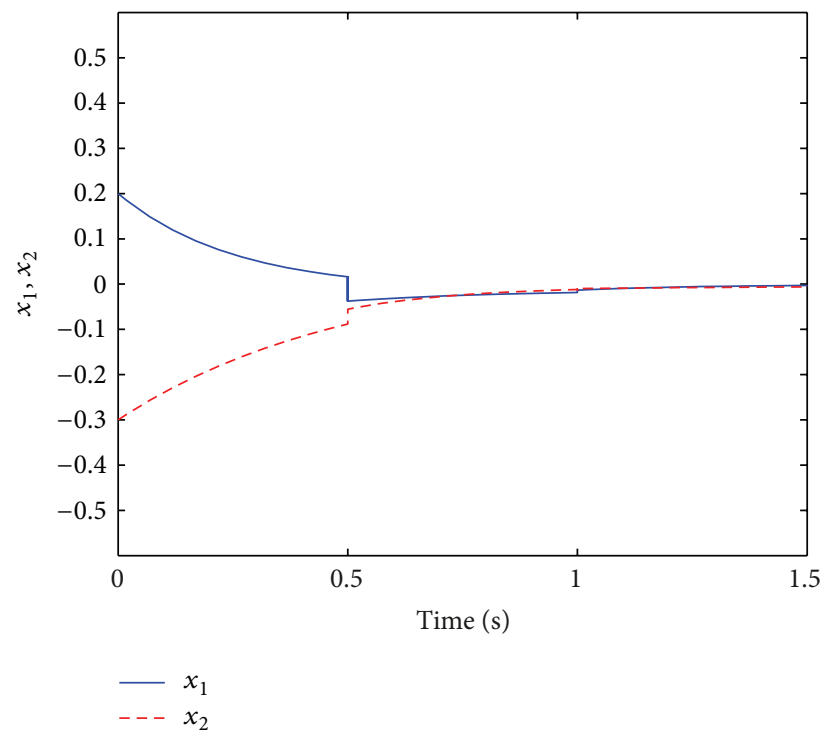

FIGURE 3: State response of system (58) under impulsive perturbations

continuous dynamics without impulse are $p$-moment stable. Applying the derived results to a class of stochastic systems with arbitrarily large delays, the deduced new stability criteria can relax some restrictions on impulses imposed by the existing results. Two illustrative examples have been provided to demonstrate the main theoretical analysis.

\section{Acknowledgments}

This work was supported by National Natural Science Foundation of China (61273091, 61104007), Natural Science Foundation of Shandong Province (ZR2011FM033), the Young and Middle-Aged Scientists Research Foundation of Shandong Province (BS2011DX013, BS2012SF008), and Taishan Scholar Project of Shandong Province of China.

\section{References}

[1] X. Mao, Stochastic Differential Equations and Applications, Horwood, Chichester, UK, 1997.

[2] B. Øksendal, Stochastic Differential Equations, Springer, New York, NY, USA, 1995.

[3] X. Mao, "Razumikhin-type theorems on exponential stability of stochastic functional-differential equations," Stochastic Processes and Their Applications, vol. 65, no. 2, pp. 233-250, 1996.

[4] X. Mao, "Stability of stochastic differential equations with Markovian switching," Stochastic Processes and Their Applications, vol. 79, no. 1, pp. 45-67, 1999.

[5] L. Huang, X. Mao, and F. Deng, "Stability of hybrid stochastic retarded systems," IEEE Transactions on Circuits and Systems I, vol. 55, no. 11, pp. 3413-3420, 2008.

[6] L. Huang and F. Deng, "Razumikhin-type theorems on stability of neutral stochastic functional differential equations," IEEE Transactions on Automatic Control, vol. 53, no. 7, pp. 1718-1723, 2008.
[7] L. Huang and X. Mao, "On input-to-state stability of stochastic retarded systems with Markovian switching," IEEE Transactions on Automatic Control, vol. 54, no. 8, pp. 1898-1902, 2009.

[8] S. Janković, J. Randjelović, and M. Jovanović, "Razumikhintype exponential stability criteria of neutral stochastic functional differential equations," Journal of Mathematical Analysis and Applications, vol. 355, no. 2, pp. 811-820, 2009.

[9] J. Luo, "Exponential stability for stochastic neutral partial functional differential equations," Journal of Mathematical Analysis and Applications, vol. 355, no. 1, pp. 414-425, 2009.

[10] G. Zong, L. Hou, and H. Yang, "Further results concerning delay-dependent $H_{\infty}$ control for uncertain discrete-time systems with time-varying delay," Mathematical Problems in Engineering, vol. 2009, Article ID 732181, 24 pages, 2009.

[11] M. Mariton, Jump Linear Systems in Automatica Control, Marcel Dekker, New York, NY, USA, 1990.

[12] H. Gao, Z. Fei, J. Lam, and B. Du, "Further results on exponential estimates of Markovian jump systems with modedependent time-varying delays," IEEE Transactions on Automatic Control, vol. 56, no. 1, pp. 223-229, 2011.

[13] G. K. Basak, A. Bisi, and M. K. Ghosh, "Stability of a random diffusion with linear drift," Journal of Mathematical Analysis and Applications, vol. 202, no. 2, pp. 604-622, 1996.

[14] X. Mao, "Robustness of stability of stochastic differential delay equations with Markovian switching," Stability and Control, vol. 3, no. 1, pp. 48-61, 2000.

[15] L. Zhang and J. Lam, "Necessary and sufficient conditions for analysis and synthesis of Markov jump linear systems with incomplete transition descriptions," IEEE Transactions on Automatic Control, vol. 55, no. 7, pp. 1695-1701, 2010.

[16] S. Xu, T. Chen, and J. Lam, "Robust $H^{\infty}$ filtering for uncertain Markovian jump systems with mode-dependent time delays," IEEE Transactions on Automatic Control, vol. 48, no. 5, pp. 900907, 2003.

[17] G. L. Wang and Q. L. Zhang, "Adaptive control of stochastic nonlinear systems with Markovian switching," International Journal of Adaptive Control and Signal Processing, vol. 26, no. 9, pp. 848-860, 2012.

[18] V. Lakshmikantham, D. D. Baĭnov, and P. S. Simeonov, Theory of Impulsive Differential Equations, World Scientific, Singapore, 1989.

[19] S. Wu, D. Han, and X. Meng, " $p$-moment stability of stochastic differential equations with jumps," Applied Mathematics and Computation, vol. 152, no. 2, pp. 505-519, 2004.

[20] Z. Liu and J. Peng, " $p$-moment stability of stochastic nonlinear delay systems with impulsive jump and Markovian switching," Stochastic Analysis and Applications, vol. 27, no. 5, pp. 911-923, 2009. 


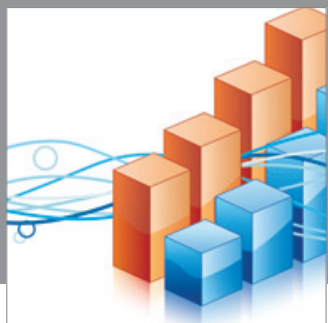

Advances in

Operations Research

mansans

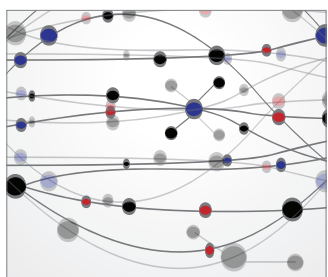

The Scientific World Journal
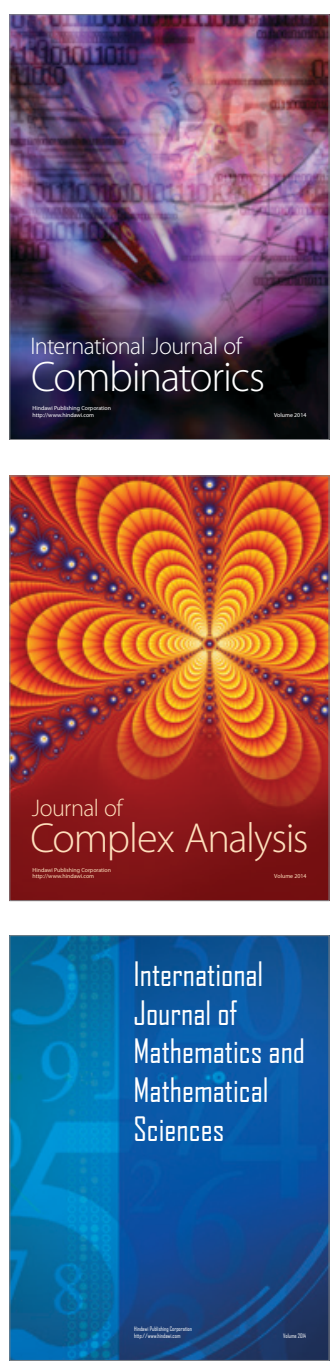
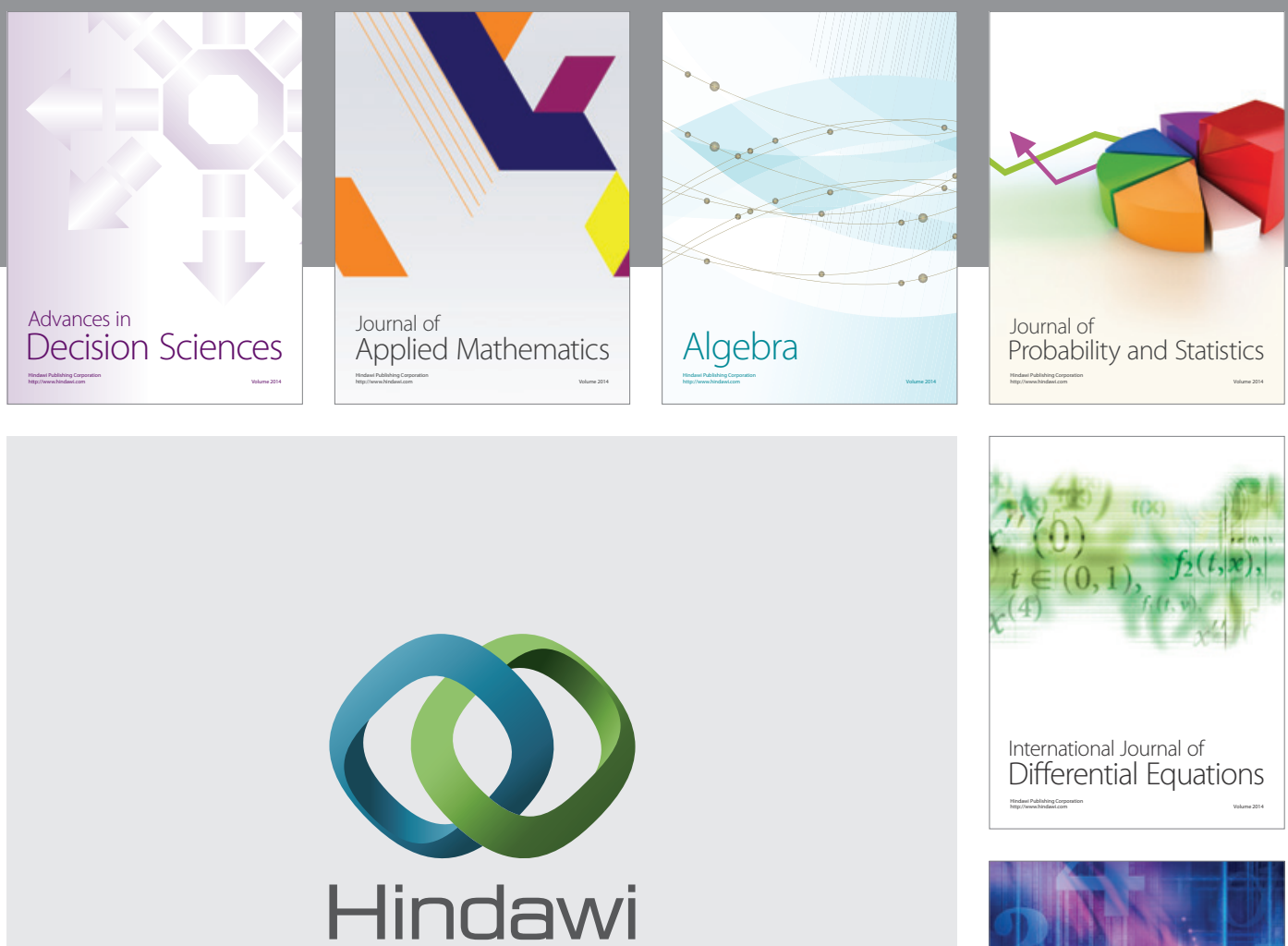

Submit your manuscripts at http://www.hindawi.com
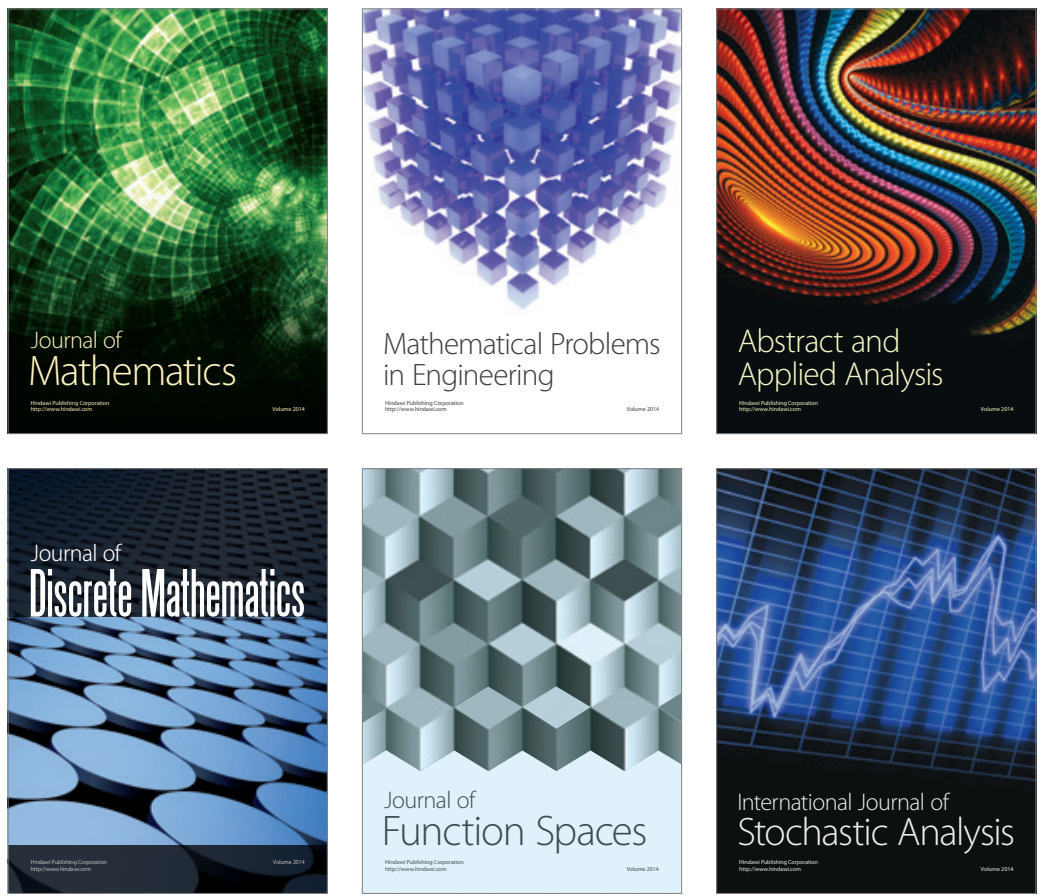

Journal of

Function Spaces

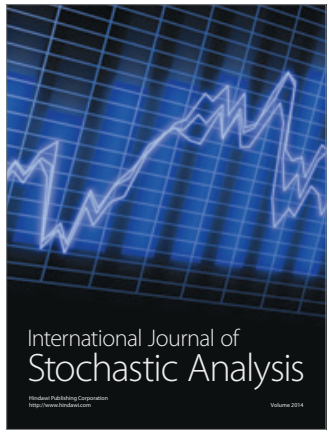

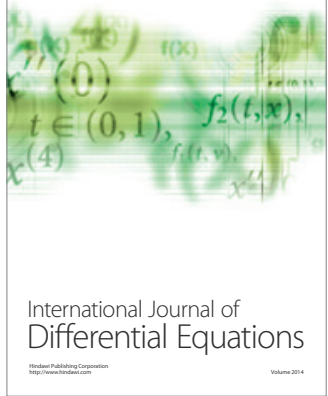
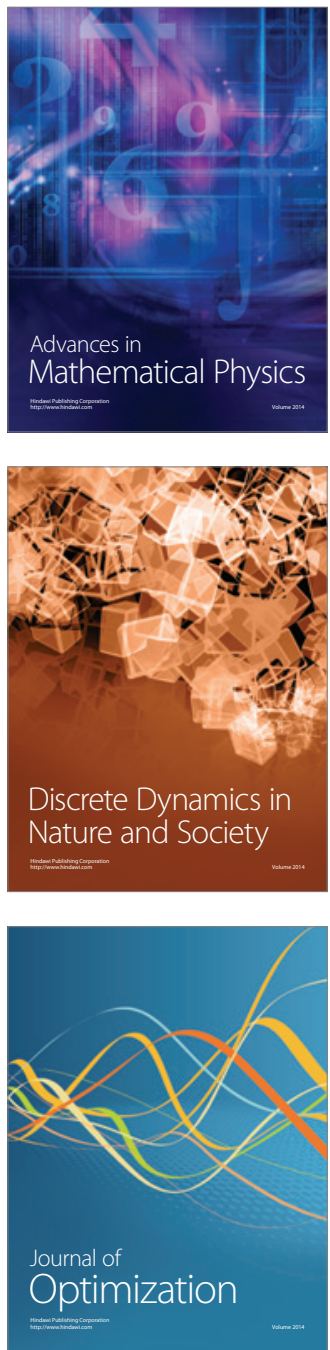